S1 Appendix: Mean-field analysis for the distributed strategy soft control performance in the Vicsek model Caiyun Wang ${ }^{1,2}$, Jing Han ${ }^{1,2^{*}}$

1 LSC, Academy of Mathematics and Systems Science, Chinese Academy of Sciences, Beijing 100190, China

2 School of Mathematical Sciences, University of Chinese Academy of Sciences, Beijing 100049, China

*hanjing@amss.ac.cn

\title{
Fixed-heading-shill scenario
}

The updating rule (without the noise) of the heading of normal agents, i.e.,

$$
\theta_{k}(t+1)=\arctan \left(\frac{\sum_{j \in N_{k}(t)} \sin \left(\theta_{j}(t)\right)}{\sum_{j \in N_{k}(t)} \cos \left(\theta_{j}(t)\right)}\right), \text { for } 1 \leq k \leq n
$$

can be written as the following form:

$$
e^{i \theta_{k}(t+1)}=\frac{\sum_{j \in N_{k}(t)} e^{i \theta_{j}(t)}}{\| \sum_{j \in N_{k}(t)} e^{i \theta_{j}(t) \|}} \text { for } k=1, \cdots, n,
$$

where $i$ is the imaginary unit. Suppose each normal agent has $\pi r^{2} \rho_{n}$ normal agent neighbors and $\pi r^{2} \rho_{s}$ shill neighbors, where $\rho_{n}$ is the density of normal agents and $\rho_{s}$ is the density of shills. Therefore, for normal agent $k$, we have:

$$
e^{i \theta_{k}(t+1)} \approx \frac{\pi r^{2} \rho_{n} e^{i \theta_{k}(t)}+\pi r^{2} \rho_{s} e^{i \theta_{s}}}{\left\|\pi r^{2} \rho_{n} e^{i \theta_{k}(t)}+\pi r^{2} \rho_{s} e^{i \theta_{s}}\right\|} .
$$

Furthermore,

$$
e^{i\left(\theta_{k}(t+1)-\theta_{s}\right)} \approx \frac{\pi r^{2} \rho_{n} e^{i\left(\theta_{k}(t)-\theta_{s}\right)}+\pi r^{2} \rho_{s}}{\left\|\pi r^{2} \rho_{n} e^{i \theta_{k}(t)}+\pi r^{2} \rho_{s} e^{i \theta_{s}}\right\|} .
$$

It follows from Eq (4) and the Euler's formula that

$$
\begin{aligned}
& \cos \left(\theta_{k}(t+1)-\theta_{s}\right)+i \sin \left(\theta_{k}(t+1)-\theta_{s}\right) \\
& \approx \frac{\pi r^{2} \rho_{n} \cos \left(\theta_{k}(t)-\theta_{s}\right)+\pi r^{2} \rho_{s}+i \sin \left(\theta_{k}(t)-\theta_{s}\right)}{\sqrt{\pi^{2} r^{4} \rho_{n}{ }^{2}+\pi^{2} r^{4} \rho_{s}{ }^{2}+2 \pi^{2} r^{4} \rho_{n} \rho_{s} \cos \left(\theta_{k}(t)-\theta_{s}\right)}} .
\end{aligned}
$$

According to the nature of the imaginary, we have:

$$
\cos \left(\theta_{k}(t+1)-\theta_{s}\right) \approx \frac{\pi r^{2} \rho_{n} \cos \left(\theta_{k}(t)-\theta_{s}\right)+\pi r^{2} \rho_{s}}{\sqrt{\pi^{2} r^{4} \rho_{n}{ }^{2}+\pi^{2} r^{4} \rho_{s}{ }^{2}+2 \pi^{2} r^{4} \rho_{n} \rho_{s} \cos \left(\theta_{k}(t)-\theta_{s}\right)}} .
$$


Furthermore,

$$
\begin{aligned}
1- & \cos ^{2}\left(\theta_{k}(t+1)-\theta_{s}\right) \\
& \approx \frac{\pi r^{2} \rho_{n}{ }^{2}}{\pi r^{2} \rho_{n}{ }^{2}+\pi r^{2} \rho_{s}{ }^{2}+2 \pi r^{2} \rho_{n} \pi r^{2} \rho_{s} \cos \left(\theta_{k}(t)-\theta_{s}\right)}\left[1-\cos ^{2}\left(\theta_{k}(t)-\theta_{s}\right)\right] \\
& =\frac{\rho_{n}^{2}}{\rho_{n}^{2}+\rho_{s}^{2}+2 \rho_{n} \rho_{s} \cos \left(\theta_{k}(t)-\theta_{s}\right)}\left[1-\cos ^{2}\left(\theta_{k}(t)-\theta_{s}\right)\right]
\end{aligned}
$$

For convenience, let $\zeta(t)=1-\cos ^{2}\left(\theta_{k}(t)-\theta_{s}\right)$. Then, when $\left|\theta_{0}-\theta_{s}\right|<\pi / 2$, direct calculations show

$$
\frac{\rho_{n}^{2}}{\rho_{n}^{2}+\rho_{s}^{2}+2 \rho_{n} \rho_{s}} \zeta(t)<\zeta(t+1)<\frac{\rho_{n}^{2}}{\rho_{n}^{2}+\rho_{s}^{2}+2 \rho_{n} \rho_{s} \cos \left(\theta_{0}-\theta_{s}\right)} \zeta(t) .
$$

Furthermore,

$$
\left(\frac{\rho_{n}^{2}}{\rho_{n}^{2}+\rho_{s}^{2}+2 \rho_{n} \rho_{s}}\right)^{t+1} \zeta(0)<\zeta(t+1)<\left(\frac{\rho_{n}^{2}}{\rho_{n}^{2}+\rho_{s}^{2}+2 \rho_{n} \rho_{s} \cos \left(\theta_{0}-\theta_{s}\right)}\right)^{t+1} \zeta(0) .
$$

Let $A=\frac{\rho_{n}^{2}}{\rho_{n}^{2}+\rho_{s}^{2}+2 \rho_{n} \rho_{s} \cos \left(\theta_{0}-\theta_{s}\right)}$ and $B=\frac{\rho_{n}^{2}}{\rho_{n}^{2}+\rho_{s}^{2}+2 \rho_{n} \rho_{s}}$, through equation (9), the convergent time step $T$ satisfies ${ }^{1}$ :

$$
\log _{A} \frac{1-\cos ^{2}\left(10^{-4}\right)}{1-\cos ^{2}\left(\theta_{0}-\theta_{s}\right)}<T<\log _{B} \frac{1-\cos ^{2}\left(10^{-4}\right)}{1-\cos ^{2}\left(\theta_{0}-\theta_{s}\right)}
$$

Therefore,

$$
\max T-\min T=\left(\frac{1}{\ln B}-\frac{1}{\ln A}\right) \ln \frac{1-\cos ^{2}\left(10^{-4}\right)}{1-\cos ^{2}\left(\theta_{0}-\theta_{s}\right)}
$$

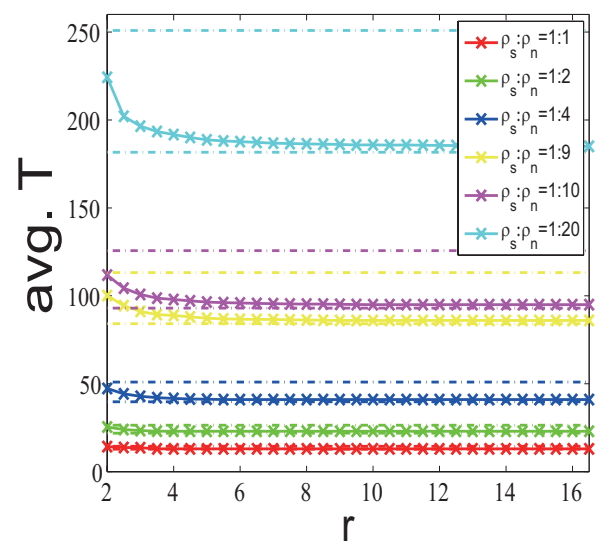

Fig 1. Simulation results v.s. mean-field results for different density ratios between shills and normal agents in the fixed-heading scenario. $\rho_{n}=1, l=25, v=0.03, \eta=0, \theta_{0}=0$ and $\theta_{s}=\pi / 4$. Dotted lines represent corresponding upper and lower bounds calculated by mean-field analysis. The simulation results are measured as the average of 100 runs on random position distributions of normal agents ${ }^{1}$.

Figure 1 shows the comparison between simulation results and mean-field results for different density ratios between shills and normal agents in the fixed-heading scenario. The

\footnotetext{
${ }^{1}$ The system without noise is regarded as reaching consensus when $\max _{1 \leq k \leq \tilde{n}} \theta_{k}(t)-\min _{1 \leq k \leq \tilde{n}} \theta_{k}(t) \leq 10^{-4}$.
} 
corresponding numerical values are given in Table 1. In general, the mean-field results conform to the simulation results especial for the lower bounds. And $\max T-\min T$ is relatively small. It proves that $r$ does not have significant influence on the soft control performance in the fixed-heading scenario.

Table 1. Simulation values v.s. mean-field value

\begin{tabular}{|c|c|c|c|c|c|c|c|c|c|}
\hline & \multicolumn{3}{|c|}{ simulation results } & \multicolumn{3}{|c|}{ mean-field results } & \multicolumn{3}{|c|}{ simulation results : mean-field results } \\
\hline$\rho_{s}: \rho_{n}$ & upper bound & lower bound & range & upper bound & lower bound & range & upper bound & lower bound & range \\
\hline $1: 1$ & 13 & 14.27 & 1.27 & 12.78 & 14.44 & 1.65 & 0.99 & 1.02 & 0.77 \\
\hline $1: 2$ & 22.96 & 25.39 & 2.43 & 21.86 & 26.40 & 4.54 & 0.96 & 1.05 & 0.53 \\
\hline $1: 4$ & 41 & 47.29 & 6.29 & 39.72 & 50.96 & 11.23 & 0.92 & 1.03 & 0.56 \\
\hline $1: 9$ & 86 & 100.12 & 14.12 & 84.13 & 113.23 & 29.10 & 0.88 & 1.02 & 0.49 \\
\hline $1: 10$ & 95 & 111.8 & 16.8 & 93.00 & 125.73 & 32.73 & 0.89 & 1.02 & 0.51 \\
\hline
\end{tabular}

\section{Evolvable-heading-shill scenario}

Similarly, for each normal agent, suppose it has $\pi r^{2} \rho_{n}$ normal agent neighbors and $\pi r^{2} \rho_{s}$ shill neighbors. Through the updating rule (without noise) of the heading of normal agents, i.e., Eq 1, we have

$$
\begin{aligned}
\theta_{k}(1) & \approx \arctan \frac{\pi r^{2} \rho_{n} \sin \theta_{0}+\pi r^{2} \rho_{s} \sin \theta_{s}}{\pi r^{2} \rho_{n} \cos \theta_{0}+\pi r^{2} \rho_{s} \cos \theta_{s}} \\
& =\arctan \frac{\rho_{n} \sin \theta_{0}+\rho_{s} \sin \theta_{s}}{\rho_{n} \cos \theta_{0}+\rho_{s} \cos \theta_{s}}
\end{aligned}
$$

Therefore,

$$
\Delta \theta \approx \arctan \frac{\rho_{n} \sin \theta_{0}+\rho_{s} \sin \theta_{s}}{\rho_{n} \cos \theta_{0}+\rho_{s} \cos \theta_{s}}
$$

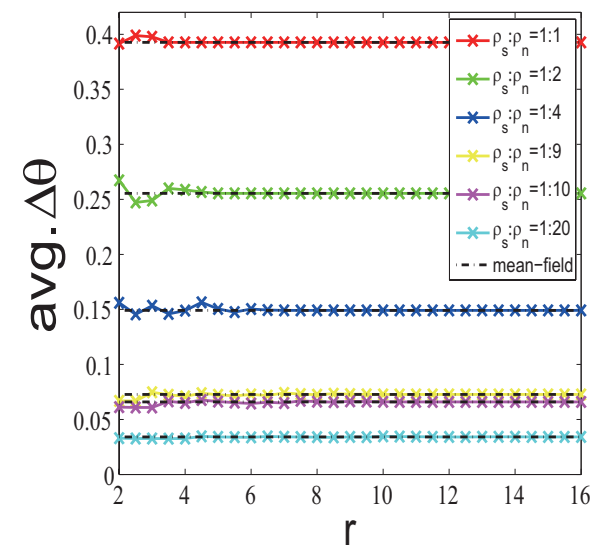

Fig 2. Simulation results v.s. mean-field results for different density ratios between shills and normal agents in the evolvable-heading scenario. $\rho_{n}=1, l=25, v=0.03, \eta=0$, $\theta_{0}=0$ and $\theta_{s}=\pi / 4$. The simulation results are measured as the average of 100 runs on random position distributions of normal agents.

Figure 2 shows the result of comparisons between simulation results and corresponding mean-field results for different density ratios between shills and normal agents in the evolvable-heading scenario. Mean-field results are roughly in accordance with simulation 
results especially. So it proves that $r$ does not have significant influence on the soft control performance in the evolvable-heading scenario.

We can see from Fig. 5D of the main text that: for $\theta_{s}>\pi / 2$, increasing $\theta_{s}$ decreases $\Delta \theta$. This means a larger perturbation perturbs less the system. We will give mean-field proof for this counter-intuitive phenomena in the following.

By Eq (13), regard $\Delta \theta$ as a function of $\theta_{s}$, then we have:

$$
\frac{\mathrm{d} \Delta \theta}{\mathrm{d} \theta_{s}}=\frac{\rho_{s}{ }^{2}+\rho_{s} \rho_{n} \cos \left(\theta_{s}-\theta_{0}\right)}{\left(\rho_{n} \sin \theta_{0}+\rho_{s} \sin \theta_{s}\right)^{2}}
$$

Therefore, when $\theta_{s}>\theta_{0}+\arccos \left(-\rho_{s} / \rho_{n}\right), \Delta \theta$ decreases with the increase of $\theta_{s}$. Especially, $\rho_{s}: \rho_{n}$ is set to be $1: 9$ in the Fig. 5D of the main text, when $\theta_{s}>\theta_{0}+\arccos \left(-\rho_{s} / \rho_{n}\right) \approx 0.5354 \pi, \Delta \theta$ decreases with the increase of $\theta_{s}$. So our mean-field proof for this counter-intuitive critical point is consistent with the simulation result. 